\title{
On Temporal Evolution in Data Streams
}

\author{
Charu C. Aggarwal \\ IBM T.J. Watson Research Center \\ 19 Skyline Drive \\ Hawthorne, NY 10532, USA \\ charu@us.ibm.com
}

\begin{abstract}
In recent years, the progress in hardware technology has made it possible for organizations to store and record large streams of transactional data. This results in databases which grow without limit at a rapid rate. This data can often show important changes in trends over time. In such cases, it is useful to understand, visualize, and diagnose the evolution of these trends. In this talk, we discuss a method to diagnose the changes in the underlying data stream and other related methods for change detection in streams. We also discuss the problem of data stream evolution in the context of mining algorithms such as clustering and classification. In many cases, mining algorithms may not function as effectively because of the change in the underlying data. We discuss the effects of evolution on mining and synopsis construction algorithms and a number of opportunities which may be available for further research on the topic.
\end{abstract}

